\title{
A NOTE ON RIGID SUBSTRUCTURES
}

\author{
R. J. PARIKH
}

\begin{abstract}
We show that a theory with a recursive set of axioms may have (nontrivial) rigid substructures and yet fail to have $\Sigma_{1}^{1}$, or $\Pi_{1}^{1}$ rigid substructures.
\end{abstract}

Let $T$ be a first order theory. By a rigid substructure for $T$, we mean a structure $R$ such that for all models $M$ of $T$, there is a unique isomorphism from $R$ into $M$. It is not required that $R$ itself be a model for $T$. $R$ is said to be a hard core for $T$ if $R$ includes all rigid substructures for $T$. (The inclusion will be unique.)

It has been pointed out by Kreisel that to every element $\alpha$ of a rigid substructure for $T$ corresponds a formula $(\exists ! x)\left(\exists x_{1}\right) \cdots\left(\exists x_{n}\right) A\left(x, \cdots, x_{n}\right)$ with $A$ quantifier free, but not necessarily vice versa. Let $F$ be the set of all such formulae and let $R$ be a subset of $F$. Miss Ville has shown (unpublished results) that $R$ corresponds to a rigid substructure of $T$ iff $R$ satisfies a particular $\Pi_{2}^{0}$ formula and hence it follows that if $T$ has a nonempty rigid substructure it has one that is $\Sigma_{1}^{1}$-recursive. On the other hand, in familiar examples, there is always a recursive $R$.

The following lemma shows that Miss Ville's result is essentially the best possible.

Lemma. Let $X,<$ be a recursive, infinitely branching tree. Then there exists a recursive theory $T$ and a recursive map from nonempty rigid substructures for $T$ to infinite branches of $X$.

Proof. $T$ is going to be roughly a description of the tree, but since we want infinite branches and only infinite branches to be rigid we must ensure that (1) no set containing an end point can be rigid; (2) no rigid set contains more than one branch; and (3) no infinite branch can be mapped into any other (i.e. our language must contain means for distinguishing one branch from another). Assume the tree described in terms

Received by the editors February 17, 1971.

AMS 1969 subject classifications. Primary 0250, 0270.

Key words and phrases. Rigid substructures, recursive theory.

(c) American Mathematical Society 1972 
of the predicates $V(x): x$ is the top vertex and $D(x, y): y$ is immediately under $x$. Let $\left\{p_{i}\right\}$ be a recursive enumeration of $X$ with $V\left(p_{1}\right)$ being true.

We expand $X$ to a larger set $X^{+}$and introduce new predicates $L$ and $S$ as follows: For each $p_{n}$ we introduce $n$ new elements $q_{n}^{1}, \cdots, q_{n}^{n}$ and extend $V$ and $L$.

(1) $V\left(q_{1}^{1}\right)$ is also true.

(2) Whenever $D\left(x, p_{n}\right)$ holds, $D\left(x, q_{n}^{i}\right)$ also holds. $D\left(q_{n}^{i}, y\right)$ never holds.

(3) $S(x, y, z)$ iff $D(x, y)$ and $D(x, z)$ and, for some $n, z$ immediately follows $y$ in the sequence $\left\langle p_{n}, q_{n}^{1}, \cdots, q_{n}^{n}, p_{n}\right\rangle$.

Call this sequence the circle $R_{n}$. Then $R_{n}$ cannot be confused with any $R_{m}, m \neq n$, but $R_{n}$ can be rotated unless a descendent of $p_{n}$ is present in which case the circle becomes rigid. This descenclent will aiso have to have descendents and so on, so to keep one point rigid, an infinite branch has to be present. (Note that $R$ rigid and $Y \subseteq R$ does not imply $Y$ rigid.)

(4) $L(x, y)$ is a pseudolinear ordering extending $D(x, y)$ i.e.

(a) $D(x, y) \rightarrow(L(x, y) \wedge\rceil L(y, x))$,

(b) $L(x, y) \wedge L(y, x) \leftrightarrow(\exists z)(\exists u)(\exists v)(S(z, u, x) \wedge S(z, v, y)) \vee(V(x) \wedge V(y))$,

(c) $L(x, y) \vee L(y, x)$

(d) $L(x, y) \wedge L(y, z) \rightarrow L(x, z)$

Note now that in terms of $V, D, S$, every $p_{n}, q_{n}^{i}$ can be defined unless $p_{n}$ has no descendents in which case only the circle $R_{n}$ can be defined. The theory $T$ consists of a description of the resulting diagram together with axioms 4(a)-4(d) above; axioms saying that for each $n>1$ there are exactly $n+1$ elements satisfying the condition

$$
R_{n}\left(x_{1}, \cdots, x_{n+1}\right) \leftrightarrow(\exists u)\left(S\left(u, x_{1}, x_{2}\right) \wedge \cdots \wedge S\left(u, x_{n+1}, x_{1}\right)\right) ;
$$

and exactly two $x$ such that $V(x)$.

To each subset $Y$ of $X$ corresponds $Y^{+} \subseteq X^{+}$defined by $Y^{+}=$ $\bigcup\left\{R_{n}: p_{n} \in Y\right\}$.

Now axioms $4\left(\right.$ a) -4 (d) completely give $L$ on any $B^{+}$where $B$ is a branch in $X$. But over larger sets, problems of comparison will arise. Hence for every rigid $R, \exists B$ such that $R \subseteq B^{+}$. Also, $B$ must be infinite and $B \subseteq R$. On the other hand $B^{+}$itself is clearly rigid. Hence $T$ has rigid substructures $R$ and $R \rightarrow R \cap X$ maps the substructures onto infinite branches of $X$. This proves the lemma.

Now we can take a tree $X, \prec$ which has no $\Pi_{1}^{1}$ or $\Sigma_{1}^{1}$ infinite branches, though it does have branches recursive in $\Sigma_{1}^{1}$. The corresponding $T$ will have no $\Pi_{1}^{1}$ or $\Sigma_{1}^{1}$ rigid substructures.

If $L$ is dropped then $T$ will have a hard core, namely $\bigcup\left\{B^{+}: B\right.$ is an infinite branch\}, which is $\Sigma_{1}^{1}$. However, there will still be no hyperarithmetic rigid substructures.

The above results can be relativised to a set $A \subseteq N$ using " $\Pi_{1}^{1}$ in $A$ " etc. 


\section{BIBLIOGRAPHY}

1. G. Kreisel, Model-theoretic invariants: Applications to recursive and hyperarithmetic operations, Theory of Models (Proc. 1963 Internat. Sympos, Berkeley), NorthHolland, Amsterdam, 1965, pp. 190-205, especially p. 193. MR 33 \#7257.

2. H. Rogers, Jr., Theory of recursive functions and effective computability, McGrawHill, New York, 1967. MR 37 \#61.

3. F. Ville, Complexité des structures rigidement contenues dans une théorie du premier ordre, C. R. Acad. Sci. Paris Sér. A 272 (1971), 561-563.

Department of Mathematics, Boston University, Boston, Massachusetts 02215 